\title{
A preliminary account of the fly fauna in Jabal Shada al-A'la Nature Reserve, Saudi Arabia, with new records and biogeographical remarks (Diptera, Insecta)
}

\author{
Magdi S. El-Hawagry', Mahmoud S. Abdel-Dayem ${ }^{1,2}$, \\ Ali A. Elgharbawy', Hathal M. Al Dhafer ${ }^{2}$
}

I Entomology Department, Faculty of Science, Cairo University, Giza 12613, Egypt 2 Plant Protection Department, College of Food and Agriculture Sciences, King Saud University, Riyadh 11451, PO Box 2460, Kingdom of Saudi Arabia

Corresponding author: Magdi S. El-Hawagry (elhawagry@gmail.com)

Academic editor: T. Dikow | Received 17 July 2016 | Accepted 9 November 2016 | Published 24 November 2016

http://zoobank.org/A09BEDD8-A29C-4885-AF8D-A250B26B073D

Citation: El-Hawagry MS, Abdel-Dayem MS, Elgharbawy AA, Dhafer HM (2016) A preliminary account of the fly fauna in Jabal Shada al-A'la Nature Reserve, Saudi Arabia, with new records and biogeographical remarks (Diptera, Insecta). ZooKeys 636: 107-139. doi: 10.3897/zookeys.636.9905

\begin{abstract}
The first list of insects of Al-Baha Province, Kingdom of Saudi Arabia (KSA) was published in 2013 and contained a total of 582 species; an addendum to this list was published in 2015 adding 142 species and bringing the total number recorded from the province to 724 insect species representing 17 orders. The previous two studies excluded Jabal Shada al-A'la Nature Reserve (SANR), so the present study in SANR, as belonging to Al-Baha Province, are complementary to the previous two. The present study presents a preliminary list of Diptera (Insecta) in SANR, with remarks on their zoogeography, and is the first of a series of planned ecological and systematic studies on different insect orders as one of the outputs of a project proposed to study the entire insect fauna of SANR.

A total number of 119 Diptera species belonging to 87 genera, 31 tribes, 42 subfamilies, and representing 30 families has been recorded from SANR in the present study. Some species have been identified only to the genus level and listed herein only because this is the first time to record their genera in KSA. Fourteen of the species are recorded for the first time for KSA, namely: Forcipomyia sahariensis Kieffer, 1923 [Ceratopogonidae]; Chaetosciara sp. [Sciaridae]; Neolophonotus sp.1; Neolophonotus sp.2; Promachus sinaiticus Efflatoun, 1934; Saropogon longicornis (Macquart, 1838); Saropogon sp. [Asilidae]; Spogostylum tripunctatum (Pallas in Wiedemann, 1818) [Bombyliidae]; Phycus sp. [Therevidae]; Hemeromyia sp.; Meoneura palaestinensis Hennig, 1937 [Carnidae]; Desmometopa inaurata Lamb, 1914 [Milichiidae]; Stomoxys niger Macquart, 1851 [Muscidae]; and Sarcophaga palestinensis (Lehrer, 1998) [Sarcophagidae].
\end{abstract}

Copyright Magdi S. El-Hawagry et al. This is an open access article distributed under the terms of the Creative Commons Attribution License (CC BY 4.0), which permits unrestricted use, distribution, and reproduction in any medium, provided the original author and source are credited. 
Zoogeographic affinities of recorded fly species suggest a closer affiliation to the Afrotropical region (46\%) than to the Palearctic region $(23.5 \%)$ or the Oriental region $(2.5 \%)$. This supports the previous studies' conclusions and emphasizes the fact that parts of the Arabian Peninsula, including Al-Baha Province, ought to be a part of the Afrotropical Region rather than of the Palaearctic Region or the Eremic Zone.

\section{Keywords}

Afrotropical, Al-Baha Province, Al-Sarah, Al-Sarawat Mountains, Arabian Peninsula, Eremic Zone, fly species, new records, Palaearctic, Tihama

\section{Introduction}

Al-Baha Province (Fig. 1) is situated in the south-western part of the Kingdom of Saudi-Arabia (KSA) between the Holy Makkah and Asir provinces. It is the smallest province in KSA (approximately $10,362 \mathrm{~km} 2$ ), situated at $41-42^{\circ} \mathrm{E}$ and $19-20^{\circ} \mathrm{N}$. It is characterized by natural tree cover (El-Juhany and Aref 2013) and agricultural plateaus. Huge and steep rocky mountains divide the province into two main sectors, a mountainous area known as 'Al-Sarat' or 'Al-Sarah' with an elevation of 1500-2450 $\mathrm{m}$ above sea level at the east forming a part of Al-Sarawat Mountains range, and a lowland coastal plain in the west, known as 'Tihama'. The second sector, Tihama, is divided into two districts, Al-Mekhwa and Qelwa (Alahmed et al. 2010, El-Hawagry et al. 2013, 2015). Jabal Shada al-A'la Nature Reserve (SANR) lies between latitudes $19.8149 \mathrm{~N}-19.8763 \mathrm{~N}$ and longitudes $41.2855 \mathrm{E}-41.3501 \mathrm{E}$ (Fig. 1). It is an isolated granite mountain massif made up of jagged spires and pinnacles, located in Al-Mekhwa district, $20 \mathrm{~km}$ to the south-west of Al-Mekhwa city, the capital of the district. It is a dissonant of the Sarawat Escarpment in the foothills of Tihama, measuring 68.62 square kilometers. Its location and its altitudinal range from 490 to 2,222 meters above sea level ensures high rainfall, a wide range of micro-climates, and a high level of biological diversity (SWA 2016).

In the lowland coastal plain, Tihama, the climate is hot in summer, warm in spring and mild in winter, with less than $100 \mathrm{~mm}$ of annual rainfall. In the mountainous area, Al-Sarah, the weather is generally cooler due to high altitude, in addition to the formation of clouds and fog accompanied by thunderstorms in winter, with a rainfall average of $405 \mathrm{~mm}$ annually (Ibrahim and Abdoon 2005; El-Hawagry and Al Dhafer 2015). The climate in SANR is intermediate between the climates in these two sectors, with a rainfall average of approximately $200 \mathrm{~mm}$ annually (Fig. 2).

SANR, as an isolated mountain massif, supports an exceptionally rich flora; with approximately 500 plant species recorded, including 63 key plant taxa including endemics and Afrotropical relicts, it is the site of highest botanical diversity known in Saudi Arabia. The exceptional floral diversity of SANR, together with the presence of griffon vultures and endemic birds of the southwestern mountains and carnivores such as, the Arabian red fox [Vulpes vulpes arabica Thomas, 1902], Arabian caracal [Caracal caracal schmitzi (Matschie, 1912)], striped hyaena [Hyaena hyaena sultana (Pocock, 


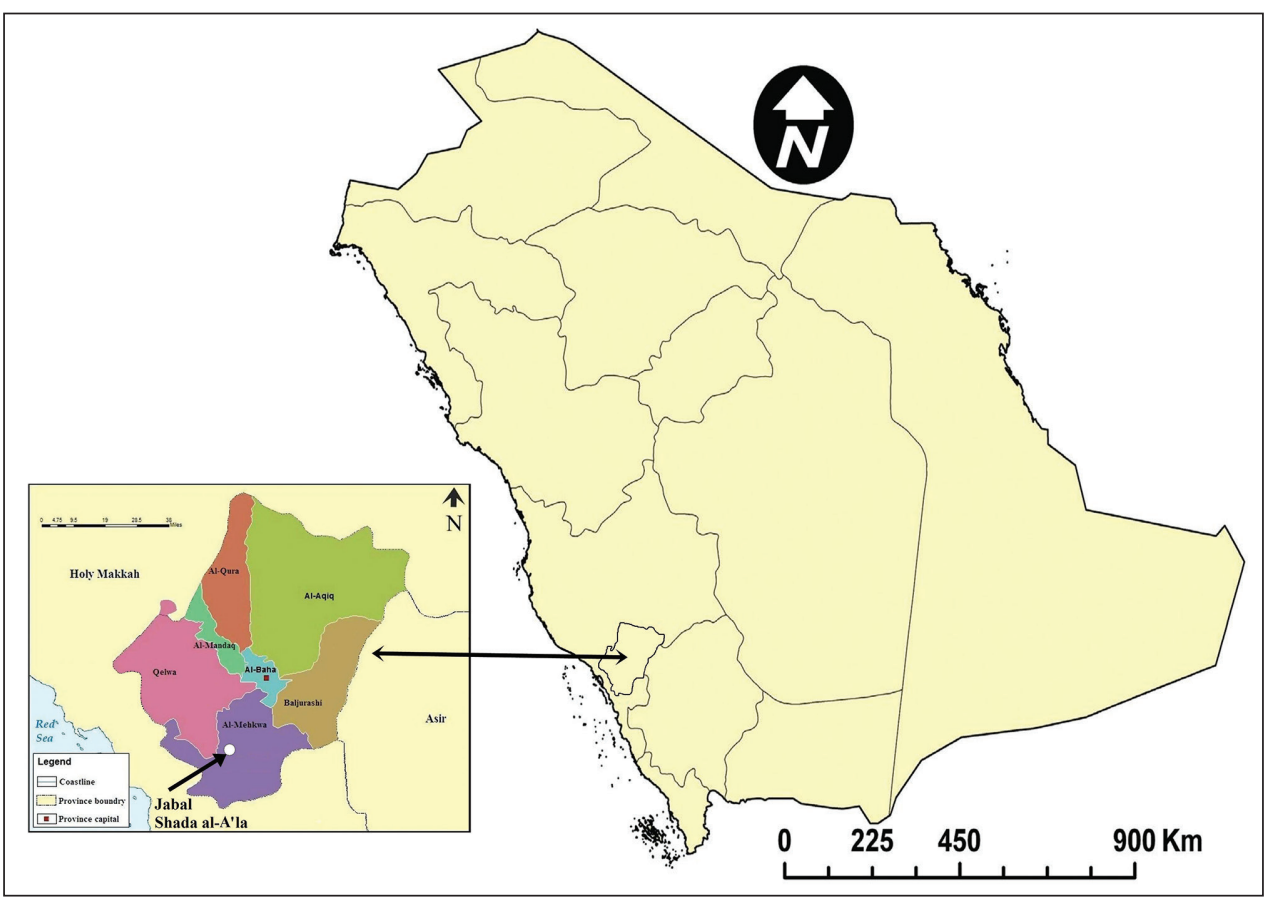

Figure I. Map of Saudi Arabia showing Al-Baha Province and Jabal Shada al-A'la Nature Reserve.

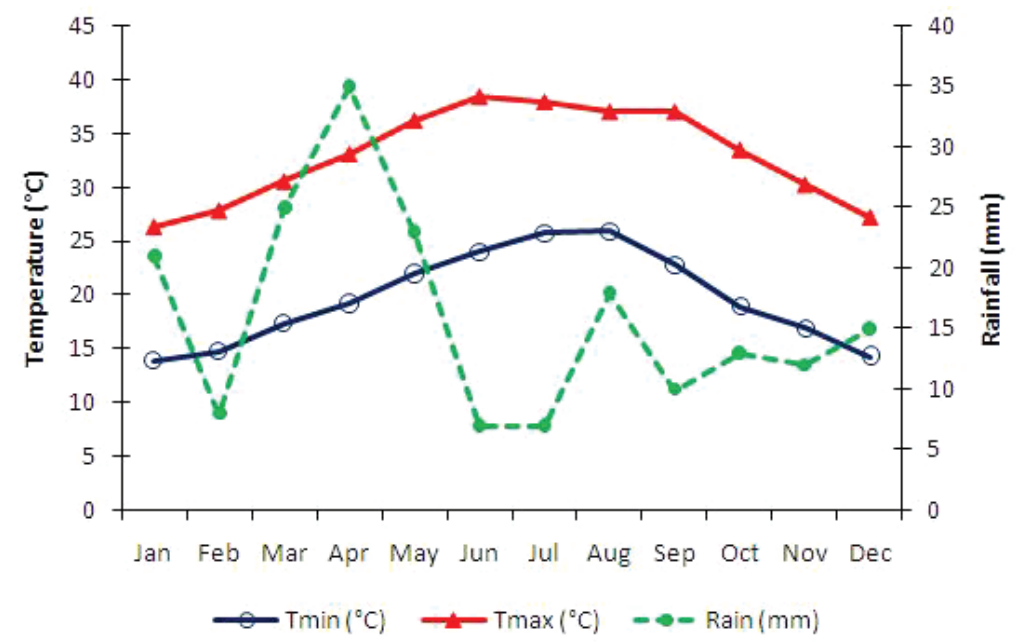

Figure 2. Monthly average temperatures and rainfall in 50 years (1950-2000). In Jabal Shada al-A'la Nature Reserve (Worldclim database: http://www.worldclim.org/).

1934)], Arabian wolf [Canis lupus arabs Pocock, 1934], sand cat [Felis margarita harrisoni Hemmer, Grubb \& Groves, 1976], and reportedly the Arabian leopard [Panthera pardus nimr Hemprich \& Ehrenberg, 1833], makes this small protected area a unique 
treasure of biological diversity. Small communities on the mountain grow a distinctive variety of coffee and other crops in terraced fields (El-Hawagry et al. 2013; SWA 2016; UAEinteract 2016).

The purpose of this paper is to present a preliminary list of Diptera (Insecta) in SANR, Al-Baha Province, KSA, with remarks on their zoogeography. This is not the final list of Diptera that occur at SANR with the study serving as a basis for further investigations as many additional collected species are still unidentified and further studies are planned to be carried out at SANR. Also, this is the first of a series of planned ecological and systematic studies on different insect orders as one of the outputs of a project proposed to study the entire insect fauna of SANR.

El-Hawagry et al. $(2013,2015)$ studied the insect fauna of Al-Baha Province excluding SANR, so the present study and other future studies in SANR are complementary to the previous two studies. Studies on the fauna of SANR are of particular interest as this area lies in a part of the Arabian Peninsula which is thought by many authors to touch three of the main zoogeographical regions: the Palaearctic, the Afrotropical, and the Oriental (Hölzel 1998).

The Afrotropical Region is supposed to encompass all of Africa south of the Sahara, with the island of Madagascar and the nearby smaller islands. Many authors add parts of the Arabian Peninsula to the Afrotropical Region as well, but there seems to be no agreement as to how much (El-Hawagry et al. 2015). This may be deduced from the fact that the south-western and southern parts of the Arabian Peninsula including AlBaha Province are strongly influenced by a subtropical to tropical climate with spring and summer rains (Abdullah and Al-Masroui 1998), and are thus dominated by a xeromesic tropical flora of palaeotropical origin, that in fact represents the impoverished northern part of an African flora (Ghazanfar and Fisher 1998; Hegazy et al. 1998). Examples of plant species with this conspicuous distribution pattern, linking southwest Arabia with the other side of the Red Sea, and commonly represented in SANR are: Barleria bispinosa (Forssk.) Vahl, Blepharis ciliaris (L.) B.L.Burtt and Hypoestes forskaolii (Vahl) R.Br. (Acanthaceae); Aloe officinalis Forssk. (Aloeaceae), Aerva javanica (Burm.f.) Juss. ex Schult., Aerva lanata (L.) A. L. Juss. ex Schultes and Celosia spp. (Amaranthaceae); Adenium obesum (Forssk.) Roem. \& Schlt. and Carissa edulis (Forssk.) Vahl (Apocynaceae); Commiphora quadricinta Schweinf. and Capparis cartilaginea Decne. (Burseraceae); Commelina forskaolii Vahl (Commelinaceae); Conyza stricta Willd., Echinops sp., Psiadia punctulata (DC.), Pulicaria undulata (DC.), Rhamnus dispermus (L.), Tagetes minuta L. and Vernonia schimperi DC. (Compositae); Sansevieria ehrenbergii Schweinf. ex Baker (Dracaenaceae); succulent Euphorbia spp. (Euphorbiaceae); Acacia asak (Forssk.), Acacia etbaica Schweinf and Indigofera spinosa Forssk. (Fabaceae); Asparagus africanus Lam. (Liliaceae); Hibiscus micranthus L. and Hibscus deflersii Schweinf. ex Cufod. (Malvaceae); Ficus ingens (Miq.) (Moraceae); Commicarpus spp. (Nyctaginaceae); Aristida adscensionis L., Cenchrus ciliaris L., Eragrostis tenella (L.) P. Beauv. ex Roemer \& Schultes and Pennisetum divisum (Gmel.) Henr. (Poaceae); Solanum incanum L. (Solanaceae); Grewia tembensis Fresen and Grewia tenax (Forssk.) (Tiliaceae); Cissus rotundifolius (Forssk.) Vahl (Vitaceae); in addition to semi-evergreen 
sclerophyllous woodlands of the Afromontane vegetation (Ghazanfar and Fisher 1998; Zohary 1973; Thomas 2016).

Sclater (1858) and Wallace (1876) proposed the classical zoogeographical regions and placed the northern border of the Afrotropics along the Tropic of Cancer, i.e. the northern limit of the Afrotropical Region was placed in Taif area, some $200 \mathrm{~km}$ north of Al-Baha Province (Hölzel 1998). Crosskey (1980) considered the northern boundaries of Yemen as the regional boundary between the Afrotropical and Palaearctic parts in the Arabian Peninsula. Extensive sampling of Insects in the Arabian Peninsula by many authors in Yemen, Oman, the United Arab Emirates and south-western mountains of KSA, have raised some interesting questions about the true extent of the Afrotropical Region in this important transitional zone. Authors indicate that Wallace's (1876) concept of the extent of the Afrotropical Arabian Peninsula is more accurate than Crosskey's (1980) limited concept of Yemen alone (Kirk-Spriggs and McGregor 2009, El-Hawagry et al. 2015). However, Uvarov (1938), Greathead (1980) and Larsen (1984) agreed that the south-western part of KSA including the study area should be united with the central Arabian deserts which are either considered as a part of the Palaearctic or by some authors as an autonomous Eremic Zone (also called the SaharoSindian faunal region).

\section{Material and methods}

Flies were collected from different localities in SANR over two successive years, 2014 and 2015 by the authors. Twelve collecting trips were made, six in 2014 in February, April, June, August, October and December, and six in 2015 in January, March, May, July, September and November. Collections were made in 6 different localities representing different altitudinal levels and habitats in SANR (Figs 13-18, Table 1). The collecting methods included sweep and aerial nets (randomly), bait traps (irregularly), light traps (6 traps, one in each locality, for one night in each trip), Malaise traps (6 traps, one in each locality, for one day in each trip), pitfall traps (90 traps, 15 in each locality, for three days in each trip), and vacuuming (one time in each locality, for 15 minutes in each trip). In addition, a few specimens were incidentally collected by hand.

All taxa are identified and arranged in alphabetical order. Dates of collection for each species are included for the purpose of mapping the activity periods of species in the study area. Each collection date is followed, between parentheses, by the method of collection used, and the latter is followed by the locality number from which the specimens are collected.

Zoogeographical affiliations of species reported in the study area were estimated using world catalogues and counted to calculate the percentage of Afrotropical, Palaearctic or Oriental elements.

Images of newly recorded species were made using a Leica MZ 125 stereo-binocular microscope (Leica Microsystems Ltd, St. Gallen, Switzerland) fitted with a digital camera (Q-imaging Micro Publisher 5.0 RTV; Zerene Systems LLC, Richland, WA, USA) at 
Table I. An overview of the collecting localities with their coordinates and common vegetation.

\begin{tabular}{|c|c|c|c|c|c|}
\hline \multirow{2}{*}{$\begin{array}{c}\text { Locality } \\
\text { no. }\end{array}$} & \multicolumn{3}{|c|}{ Coordinates (in decimal degrees) } & \multicolumn{2}{|c|}{ The most common plants in the locality } \\
\hline & Elevation (M) & Latitude (N) & Longitude $(\mathrm{E})$ & \begin{tabular}{|c|} 
Species \\
\end{tabular} & Family \\
\hline \multirow{10}{*}{1} & \multirow{10}{*}{1666} & \multirow{10}{*}{19.8429} & \multirow{10}{*}{41.3115} & Barleria bispinosa (Forssk.) & Acanthaceae \\
\hline & & & & Carissa edulis L. & Apocynaceae \\
\hline & & & & Conyza stricta Willd. & Compositae \\
\hline & & & & Psiadia punctulata (DC.) & , \\
\hline & & & & Rhamnus dispermus (L.) & , \\
\hline & & & & Aristida adscensionis $\mathrm{L}$. & Poaceae \\
\hline & & & & Acacia etbaica Schweinf & Fabaceae \\
\hline & & & & Indigofera spinosa Forssk. & , \\
\hline & & & & Hibiscus micranthus $\mathrm{L}$. & Malvaceae \\
\hline & & & & Hibscus deflersii Schweinf. ex Cufod. & , \\
\hline \multirow{14}{*}{2} & \multirow{14}{*}{1611} & \multirow{14}{*}{19.8402} & \multirow{14}{*}{41.3114} & Barleria bispinosa (Forssk.) & Acanthaceae \\
\hline & & & & $\begin{array}{l}\text { Hypoestes forskaolii (Vahl) } \\
\text { Aerva javanica (Burm.f.) }\end{array}$ & Amaranthaceae \\
\hline & & & & Capparis cartilaginea Decne. & Burseraceae \\
\hline & & & & Echinops sp. & Compositae \\
\hline & & & & Pulicaria undulata (DC.) & , \\
\hline & & & & Tagetes minuta $\mathrm{L}$. & 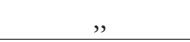 \\
\hline & & & & Vernonia schimperi DC. & , \\
\hline & & & & Cenchrus ciliaris $\mathrm{L}$. & Poaceae \\
\hline & & & & Eragrostis tenella $(\mathrm{L})$. & , \\
\hline & & & & Pennisetum divisum (Gmel.) & , \\
\hline & & & & Indigofera spinosa Forssk. & Fabaceae \\
\hline & & & & Ficus ingens (Miq.) & Moraceae \\
\hline & & & & Commicarpus spp. & Nyctaginaceae \\
\hline & & & & Solanum incanum L. & Solanaceae \\
\hline \multirow{15}{*}{3} & \multirow{15}{*}{1563} & \multirow{15}{*}{19.8388} & \multirow{15}{*}{41.3101} & Barleria bispinosa (Forssk.) & Acanthaceae \\
\hline & & & & Aerva javanica (Burm.f.) & Amaranthaceae \\
\hline & & & & Aerva lanata (L.) & , \\
\hline & & & & Asparagus africanus Lam. & Liliaceae \\
\hline & & & & Commiphora quadricinta Schweinf. & Burseraceae \\
\hline & & & & Commelina forskaolii Vahl & Commelinaceae \\
\hline & & & & Tagetes minuta $\mathrm{L}$. & Compositae \\
\hline & & & & Aristida adscensionis L. & Poaceae \\
\hline & & & & Cenchrus ciliaris $\mathrm{L}$. & , \\
\hline & & & & Eragrostis tenella $(\mathrm{L})$. & , \\
\hline & & & & Indigofera spinosa Forssk. & Fabaceae \\
\hline & & & & Solanum incanum L. & Solanaceae \\
\hline & & & & Grewia tembensis Fresen & Tiliaceae \\
\hline & & & & Grewia tenax (Forssk.) & ”, \\
\hline & & & & Cissus rotundifolius (Forssk.) & Vitaceae \\
\hline \multirow{4}{*}{4} & \multirow{4}{*}{1474} & \multirow{4}{*}{19.8452} & \multirow{4}{*}{41.3044} & Aerva javanica (Burm.f.) & Amaranthaceae \\
\hline & & & & Adenium obesum (Forssk.) & Apocynaceae \\
\hline & & & & Tagetes minuta L. & Compositae \\
\hline & & & & Cenchrus ciliaris $\mathrm{L}$. & Poaceae \\
\hline
\end{tabular}




\begin{tabular}{|c|c|c|c|c|c|}
\hline \multirow{2}{*}{$\begin{array}{c}\text { Locality } \\
\text { no. }\end{array}$} & \multicolumn{3}{|c|}{ Coordinates (in decimal degrees) } & \multicolumn{2}{|c|}{ The most common plants in the locality } \\
\hline & Elevation (M) & Latitude (N) & Longitude (E) & Species & Family \\
\hline & & & & Acacia asak (Forssk.) & Fabaceae \\
\hline & & & & Acacia etbaica Schweinf & , \\
\hline & & & & Indigofera spinosa Forssk. & , \\
\hline & & & & Solanum incanum L. & Solanaceae \\
\hline \multirow{8}{*}{5} & \multirow{8}{*}{1325} & \multirow{8}{*}{19.8511} & \multirow{8}{*}{41.3006} & Barleria bispinosa (Forssk.) & Acanthaceae \\
\hline & & & & Blepharis ciliaris (L.) & , \\
\hline & & & & Aerva javanica (Burm.f.) & Amaranthaceae \\
\hline & & & & Aerva lanata (L.) & , \\
\hline & & & & Acacia asak (Forssk.) & Fabaceae \\
\hline & & & & Acacia etbaica Schweinf & , \\
\hline & & & & Indigofera spinosa Forssk. & , \\
\hline & & & & Solanum incanum L. & Solanaceae \\
\hline \multirow{8}{*}{6} & \multirow{8}{*}{1225} & \multirow{8}{*}{19.8627} & \multirow{8}{*}{41.3015} & Barleria bispinosa (Forssk.) & Acanthaceae \\
\hline & & & & Blepharis ciliaris (L.) & , \\
\hline & & & & Aloe officinalis Forssk. & Aloeaceae \\
\hline & & & & Psiadia punctulata (DC.) & Compositae \\
\hline & & & & Sansevieria ehrenbergii Schweinf. & Dracaenaceae \\
\hline & & & & Cenchrus ciliaris $\mathrm{L}$. & Poaceae \\
\hline & & & & Acacia asak (Forssk.) & Fabaceae \\
\hline & & & & Solanum incanum L. & Solanaceae \\
\hline
\end{tabular}

the Plant Protection Department, College of Food and Agriculture Sciences, King Saud University. Photo automontage was performed by Zerene stacker program version 1.04 (Innovative Solutions, Bucharest, Romania).

Many studies and keys have been consulted in order to identify, classify and estimate the zoogeographical affiliation of collected specimens and such studies are indicated after each taxon in the list, in addition to the following: Abdullah and Merdan (1995), Amoudi (1993), Dawah and Abdullah (2006), El-Hawagry (2015), El-Hawagry and Gilbert (2014), El-Hawagry et al. (2000), Evenhuis and Greathead (2015), Greathead (1980, 1988), Londt (2008), McAlpine (1981), Pape (1996), Pape and Thompson (2016), Soós and Papp (1984-1993), Unwin (1991).

Unidentified specimens (or photos of specimens)were sent to experts for identification, as indicated after each of these taxa in the list.

Flies of suborder Nematocera were examined and preserved in alcohol, while other flies were glued to pinned stiff paper points, and all are deposited at the King Saud University Museum of Arthropods, Riyadh, Saudi Arabia (KSMA).

Abbreviations used:
AF Afrotropical
BT Bait trap
HP Hand-collecting

KSMA King Saud University Museum of Arthropods, Riyadh, Saudi Arabia 


$\begin{array}{ll}\text { LT } & \text { Light trap } \\ \text { MT } & \text { Malaise trap } \\ \text { NE } & \text { Nearctic } \\ \text { OR } & \text { Oriental } \\ \text { PA } & \text { Palaearctic } \\ \text { PT } & \text { Pitfall trap }\end{array}$

SANR Jabal Shada al-A'la Nature Reserve

SW Sweeping and areal nets

VC Vacuuming

\section{Results}

A total of 119 fly species belonging to 87 genera, 31 tribes, 42 subfamilies, and representing 30 families was recorded from SANR through the present study. Some species have been identified only to genus and listed herein as the genera were not previously recorded from KSA.

Most of the recorded fly species are characteristic of the Afrotropical region. Table (2) indicates the zoogeographic affinities of recorded species suggesting a closer affiliation to the Afrotropical region (46\%) than to the Palearctic region (23.5\%) or the Oriental region $(2.5 \%)$.

Table 2. Zoogeographic affinities of fly species of Jabal Shada al-A'la Nature Reserve (SANR).

\begin{tabular}{l|c|c}
\hline \multirow{2}{*}{ Region } & \multicolumn{2}{c}{ Affinities } \\
\cline { 2 - 3 } & No. of species & $\%$ \\
\hline Afrotropical & 55 & 46 \\
\hline Palaearctic & 28 & 23.5 \\
\hline Oriental & 3 & 2.5 \\
\hline Cosmopolitan & 14 & 12 \\
\hline Undetermined & 19 & 16 \\
\hline
\end{tabular}

\section{List of species recorded at SANR to date}

\section{Order Diptera}

Suborder Nematocera

\section{Family Bibionidae}

Dilophus tridentatus Walker, 1848

15 February 2014 (MT1), 5 May 2015 (SW1).

Identification: Haenni (1985).

Known distribution: AF. 
Family Ceratopogonidae

Subfamily Ceratopogoninae

Tribe Culicoidini

Culicoides kingi (Austen, 1912)

23 August 2014 (LT2, LT5).

Identification: Alahmed et al. (2010), Boorman (1989).

Known distribution: AF.

Subfamily Forcipomyiinae

Forcipomyia sahariensis Kieffer, 1923

23 August 2014 (LT1).

Identification: Lewanczyk et al. (2009).

Known distribution: AF. First record from KSA.

\section{Family Culicidae}

Subfamily Anophelinae

Anopheles multicolor Cambouliu, 1902

23 August 2014 (LT2), 15 February 2014 (LT3).

Identification: Glick (1992).

Known distribution: PA.

\section{Subfamily Culicinae}

Aedes caspius (Pallas, 1771)

15 February 2014 (LT1, PT4).

Identification: Alikhan et al. (2014).

Known distribution: PA.

Culex pipiens Linnaeus, 1758

23 August 2014 (PT4).

Identification: Thielman and Hunter (2007).

Known distribution: Cosmopolitan.

\section{Family Sciaridae}

Chaetosciara sp. Fig. 7

15 February 2014 (MT1), 23 August 2014 (LT2).

Remark: This seems to be the first record of Sciaridae from KSA..

Identification: Steffan (1981) and Mohrig et al. (2012).

Known distribution: Undetermined. 


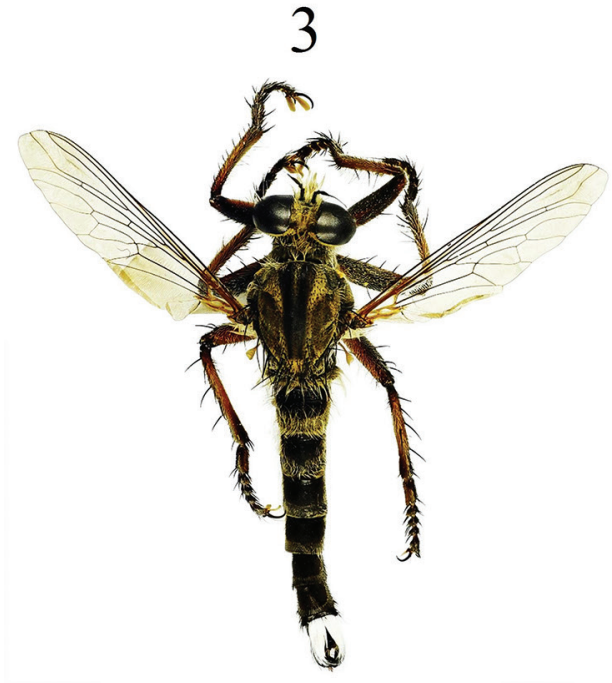

5

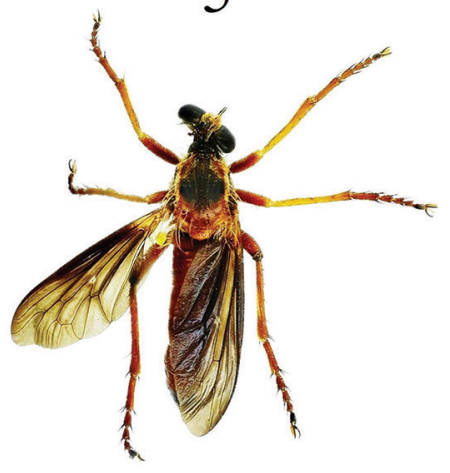

4

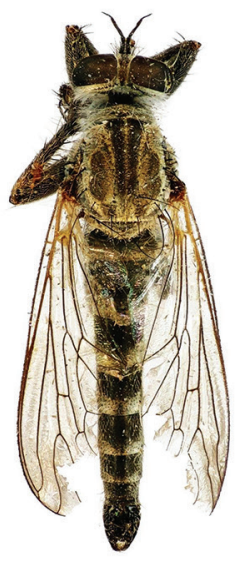

6

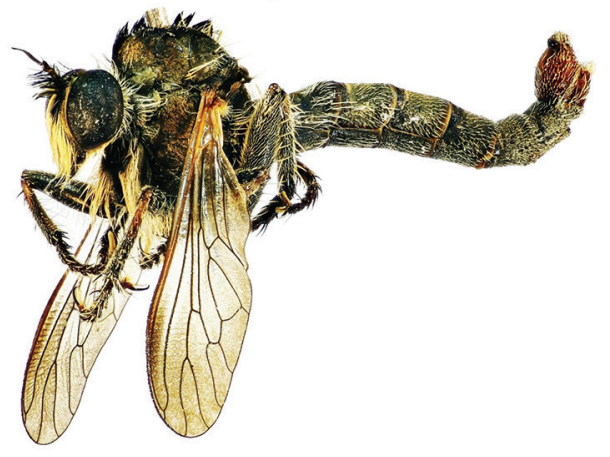

\section{7}

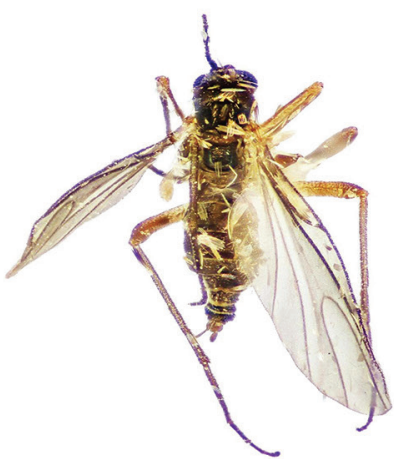

Figures 3-7. 3 Promachus sinaiticus Efflatoun 4 Neolophonotus sp.1 5 Saropogon longicornis (Macquart) 6 Neolophonotus sp. 7 Chaetosciara sp. 
Suborder Brachycera

Infraorder Asilomorpha

Superfamily Asiloidea

Family Asilidae

Subfamily Asilinae

Tribe Asilini

Neolophonotus sp1. Fig. 4

14-15 February 2014 (MT1, MT3), 21 April 2014 (LT3), 27 January 2015 (MT2, MT3, MT5), 5 May 2015 (SW1), 27 July 2015 (LT2).

Remark: This seems to be the first record of this genus from KSA.

Identification: Dr. Jason G.H. Londt, from photos (personal communication). Known distribution: Undetermined.

Neolophonotus sp2. Fig. 6

15 February 2014 (MT3), 15 November 2015 (MT3).

Remark: This seems to be the first record of this genus from KSA.

Identification: Dr. Jason G.H. Londt, from photos (personal communication).

Known distribution: Undetermined.

\section{Subfamily Apocleinae}

Promachus sinaiticus Efflatoun, 1934 Fig. 3

20 April 2014 (LT6), 3 June 2014 (LT2, MT4), 3-5 June 2014 (SW2), 15 November 2015 (MT6).

Identification: Efflatoun $(1934,1937)$.

Known distribution: PA. First record of the species from the KSA.

\section{Subfamily Dasypogoninae}

\section{Tribe Dasypogonini}

Saropogon longicornis (Macquart, 1838) Fig. 5

3 June 2014 (MT3).

Identification: Efflatoun $(1934,1937)$.

Known distribution: PA. First record from KSA.

Saropogon sp.

15 November 2015 (MT6).

Remark: This seems to be the first record of this genus from KSA.

Identification: Efflatoun (1934, 1937).

Known distribution: Undetermined.

\section{Subfamily Laphystiinae}

Trichardis leucocomus (Wulp, 1899)

3 June 2014 (MT5), 5 May 2015 (MT5).

Identification: Dr Torsten Dikow, from photos (personal communication).

Known distribution: PA. 


\section{Family Bombyliidae}

Subfamily Bombyliinae

Tribe Bombyliini

Bombylella delicata Wiedemann, 1830

5 June 2014 (SW6), 28 July 2015 (SW3).

Identification: Magdi El-Hawagry using Greathead $(1980,1988)$.

Known distribution: AF.

Bombylius pallidipilus Greathead, 1967

15 February 2014 (MT1), 23 August 2014 (LT2).

Identification: Magdi El-Hawagry using Greathead (1980, 1988).

Known distribution: AF.

Systoechus horridus Greathead, 1980

21 April 2014 (LT2), 3 May 2015 (LT5), 14 November 2015 (LT6).

Identification: Magdi El-Hawagry using Greathead (1980, 1988).

Known distribution: PA.

\section{Subfamily Anthracinae}

Tribe Anthracini

Anthrax sticticus Klug, 1832

22 April 2015 (LT).

Identification: Magdi El-Hawagry using Greathead (1980, 1988).

Known distribution: AF, PA.

Spogostylum candidum (Sack, 1909)

4 June 2014 (SW6).

Identification: Magdi El-Hawagry using Greathead $(1980,1988)$.

Known distribution: OR, PA.

Spogostylum isis (Meigen, 1820)

29 July 2015 (PT5).

Identification: Magdi El-Hawagry using Greathead (1980, 1988).

Known distribution: PA.

Spogostylum tripunctatum (Pallas in Wiedemann, 1818)

4-5 June 2014 (SW2), 2 September 2015 (LT6).

Identification: Magdi El-Hawagry using Greathead (1980, 1988).

Known distribution: PA. First record from KSA.

\section{Tribe Exoprosopini}

Defilippia nigrifimbriata (Hesse, 1956)

17 October 2014 (MT5).

Identification: Magdi El-Hawagry using Greathead (1980, 1988).

Known distribution: AF.

Exoprosopa disrupta tihamae Greathead, 1980 Fig. 9

3 June 2014 (SW1).

Identification: Magdi El-Hawagry using Greathead (1980, 1988). 

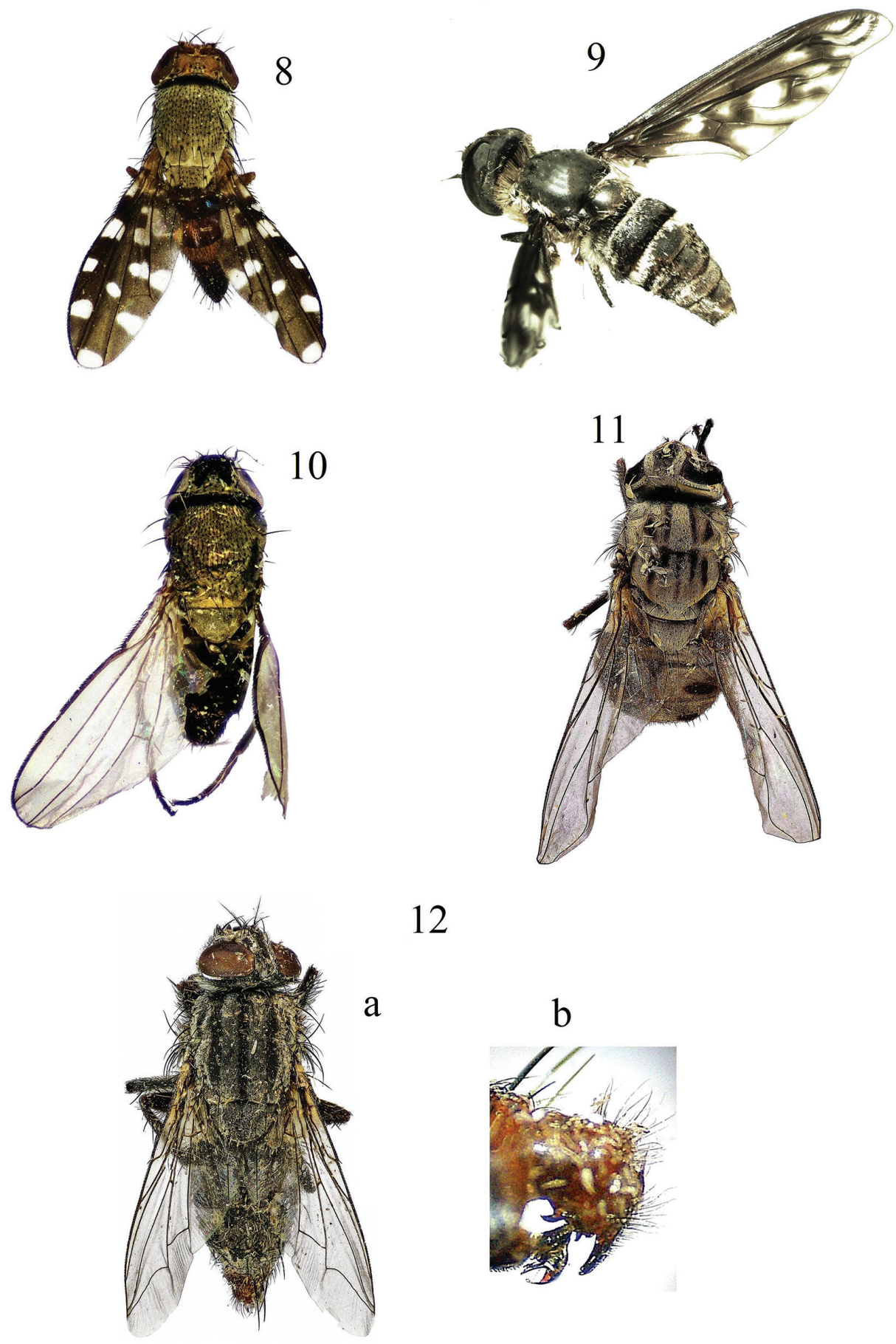

Figures 8-12.8 Actocetor margaritatus Wiedemann 9 Exoprosopa disrupta tihamae Greathead 10 Desmometopa inaurata Lamb II Stomoxys niger Macquart 12 a Sarcophaga palestinensis (Lehrer), habitus b same, male genitalia. 
Known distribution: AF.

Heteralonia bisecta Greathead, 1988

29 July 2015 (PT5).

Identification: Magdi El-Hawagry using Greathead (1980, 1988).

Known distribution: AF.

Pterobates chalybaeus (Röder, 1887)

3 November 2014 (HP6).

Identification: Magdi El-Hawagry using Greathead (1980, 1988).

Known distribution: PA.

\section{Tribe Villini}

Exhyalanthrax triangularis Bezzi, 1924

27 January 2015 (MT5), 5 May 2015 (MT2, MT4), 15 November 2015 (MT4).

Identification: Magdi El-Hawagry using Greathead (1980, 1988).

Known distribution: AF.

Pachyanthrax circe (Klug, 1832)

5 May 2015 (MT4).

Identification: Magdi El-Hawagry using Greathead (1980, 1988).

Known distribution: AF.

Villa bivirgata Austen, 1937

3 June 2014 (SW4), 5 May 2015 (SW4).

Identification: Magdi El-Hawagry using Greathead (1980, 1988) and EL-Hawagry and Greathead (2006).

Known distribution: PA.

Villa paniscoides Bezzi, 1912

3 June 2014 (SW4), 27-28 July 2015 (SW1), 15 November 2015 (MT4).

Identification: Magdi El-Hawagry using Greathead (1980, 1988) and EL-Hawagry and Greathead (2006).

Known distribution: AF.

\section{Tribe Xeramoebini}

Desmatoneura sp.

4 June 2014 (SW4).

Identification: Magdi El-Hawagry using El-Hawagry and Evenhuis (2008).

Known distribution: Undetermined.

Petrorossia albula Zaitzev, 1962

5 June 2014 (SW2), 27 July 2015 (SW1).

Identification: Magdi El-Hawagry using Greathead (1980, 1988).

Known distribution: PA.

Petrorossia letho (Wiedemann, 1828)

5 June 2014 (SW4), 27 July 2015 (SW1). 
Identification: Magdi El-Hawagry using Greathead (1980, 1988).

Known distribution: PA.

Petrorossia tropicalis Bezzi, 1921

3-5 June 2014 (SW2, SW4), 5 May 2015 (MT3), 27 July 2015 (SW4).

Identification: Magdi El-Hawagry using Greathead (1980, 1988).

Known distribution: AF.

Family Therevidae

Phycus sp.

1 June 2014 (LT5), 24 August 2014 (LT6).

Remark: This seems to be the first record of the genus from KSA.

Identification: Dr Martin Hauser (personal communication).

Known distribution: AF.

Superfamily Empidoidea

Family Dolichopodidae

Subfamily Diaphorinae

Asyndetus albifacies Parent, 1929

27 July 2015 (SW).

Identification: Grichanov (2007).

Known distribution: AF.

Subfamily Dolichopodinae

Dolichopus sp.

23 August 2014 (LT4), 10 December 2014 (LT6), 26 January 2015 (PT4), 27 July 2015 (LT6).

Identification: Grichanov (2007).

Known distribution: Undetermined.

Tachytrechus planitarsis Becker, 1907

23 August 2014 (LT2).

Identification: Grichanov (2007).

Known distribution: PA.

Superfamily Nemestrinoidea

Family Nemestrinidae

Trichopsidea costata Loew, 1858

10 December 2014 (LT6).

Identification: Narchuk (2007).

Known distribution: AF. 


\section{Superfamily Tabanoidea}

Family Tabanidae

Haematopota pluvialis (Linnaeus, 1758)

15 November 2015 (LT6).

Identification: Amoudi and Leclercq (1992) and Leclercq (1982, 1986, 2000).

Known distribution: PA.

\section{Infraorder Muscomorpha}

Section Aschiza

Superfamily Platypezoidea

Family Phoridae

Megaselia scalaris (Loew, 1866)

23 April 2014 (PT2, PT3), 5 June 2014 (PT4), 2 March 2015 (PT4), 29 July 2015

(PT5), 23 August 2015 (LT3).

Identification: Magdi El-Hawagry.

Known distribution: Cosmopolitan.

Section Schizophora

Subsection Acalyptratae

Family Carnidae

Hemeromyia sp. 23 August 2014 (LT1).

Remark: This seems to be the first record of the genus from KSA.

Identification: Sabrosky (1987).

Known distribution: Undetermined.

Meoneura palaestinensis Hennig, 1937

23 August 2014 (LT1, PT2).

Identification: Papp (1978).

Known distribution: PA.

\section{Family Chloropidae}

Subfamily Chloropinae

Pachylophus pellucidus Becker, 1910

24 August 2014 (MT6).

Identification: Deeming and Al-Dhafer (2012).

Known distribution: AF.

Thaumatomyia notata (Meigen, 1830)

27 January 2015 (LT1).

Identification: Deeming and Al-Dhafer (2012).

Known distribution: AF, PA.

\section{Subfamily Oscinellinae}

Anatrichus pygmaeus Lamb, 1918

27 July 2015 (VC5).

Identification: Deeming and Al-Dhafer (2012).

Known distribution: AF. 
Aphanotrigonum subfasciellum Collin, 1949

4 June 2014(SW4), 24 August 2014 (LT6).

Identification: Deeming and Al-Dhafer (2012).

Known distribution: PA.

Lasiochaeta vulgaris (Adams, 1905)

15 February 2014 (MT1), 8 December 2014 (VC1, VC4), 5 May 2015 (MT4).

Identification: Deeming and Al-Dhafer (2012).

Known distribution: AF.

Polyodaspis robusta (Lamb, 1918)

15 February 2014 (MT1, PT1), 17 October 2014 (LT1), 27 July 2015 (VC2).

Identification: Deeming and Al-Dhafer (2012) for genus, and Lamb (1918) for species. Known distribution: AF.

Scoliophthalmus micantipennis Duda, 1935

5 May 2015 (MT6).

Identification: Identification: Deeming and Al-Dhafer (2012).

Known distribution: AF.

Scoliophthalmus trapezoides Becker, 1903

5 May 2015 (MT6).

Identification: Identification: Deeming and Al-Dhafer (2012).

Known distribution: AF.

\section{Subfamily Siphonellopsinae}

Apotropina gregalis (Lamb, 1937)

23 August 2014 (LT5, LT6, PT2, PT3, PT4, PT5, PT6), 17 October 2014 (LT5), 8 December 2014 (VC4), 2-3 March 2015 (PT4, PT5), 17 July 2015 (LT3, MT4), 15 November 2015 (LT6).

Identification: Identification: Deeming and Al-Dhafer (2012).

Known distribution: AF.

Family Chyromyidae

Subfamily Chyromyinae

Somatiosoma eremicolum Ebejer, 2008

15 February 2014 (MT4).

Identification: Ebejer (2008).

Known distribution: AF.

\section{Family Conopidae}

Subfamily Myopinae

Tribe Zodionini

Zodion cinereum (Fabricius, 1794)

5 May 2015 (MT6).

Mei \& Stuke J-H (2008) has been consulted to identify this species.

Identification: Mei and Stuke (2008).

Known distribution: PA. 


\section{Family Diopsidae}

Diopsis apicalis Dalman, 1817

5 May 2015 (LT2, SW1).

Identification: Dawah and Abdullah (2008).

Known distribution: AF.

Sphyracephala beccarii (Rondani, 1873)

2 June 2014 (LT6), 3 June 2014 (LT3, LT4), 3 June 2014 (MT2), 27 January 2015 (LT4), 5 May 2015 (LT1, SW1), 15 November 2015 (LT6).

Identification: Dawah and Abdullah (2008).

Known distribution: AF.

\section{Family Drosophilidae}

Subfamily Drosophilinae

Tribe Drosophilini

Drosophila melanogaster Meigen, 1830

17-18 October 2014 (LT3, PT2), 8 December 2014 (PT2), 26-27 January 2015 (LT1, MT1, MT2, PT1, PT2), 2 March 2015 (PT1, PT2, PT4).

Identification: Magdi El-Hawagry.

Known distribution: Cosmopolitan.

Zaprionus indianus Gupta, 1970

2 March 2014 (PT5), 23 August 2014 (LT2), 18 October 2014 (PT1, PT2, PT4, PT5). Identification: Amoudi et al. (1991).

Known distribution: OR.

\section{Family Ephydridae}

Subfamily Discomyzinae

\section{Tribe Discomyzini}

Actocetor indicus (Wiedemann 1824)

23 April 2014 (PT4, PT5), 17 October 2014 (LT4).

Identification: Dawah and Abdullah (2006), Becker (1903) and Wiedemann (1824).

Known distribution: AF.

Actocetor margaritatus Wiedemann, 1830 Fig. 8

28 February 2014 (PT3), 23 August 2014 (PT1, PT2, PT4, PT5), 10 December (2014 (LT6), 5 May 2015 (LT4, SW1).

Identification: Dawah and Abdullah (2006), Becker (1903) and Wiedemann (1830).

Known distribution: AF.

\section{Tribe Psilopini}

Psilopa nilotica (Becker, 1903)

15 February 2014 (LT2, MT2), 4 June 2014 (SW4), 29 July 2015 (PT4, PT5).

Identification: Dawah and Abdullah (2006), Becker (1903).

Known distribution: AF, PA. 


\section{Subfamily Hydrelliinae}

Notiphila ignobilis Loew, 1862

29 July 2015 (MT6).

Identification: Dawah and Abdullah (2006), Becker (1903).

Known distribution: AF.

Family Lonchaeidae

Subfamily Lonchaeinae

Tribe Lonchaeini

Silba virescens Macquart, 1851

15 February 2014 (SW6).

Identification: MacGowan \& Friedberg (2009).

Known distribution: AF.

\section{Family Milichiidae}

Subfamily Madizinae

Desmometopa inaurata Lamb, 1914 Fig. 10

27 January 2015 (LT2), 29 July 2015 (PT4).

Identification: Deeming (1998).

Known distribution: AF. First record from KSA.

Desmometopa varipalpis Malloch, 1927

5 May 2015 (PT5), 29 July 2015 (PT6).

Identification: Identification: Deeming (1998).

Known distribution: AF.

\section{Subfamily Phyllomyzinae}

Phyllomyza sp.

15 February 2014 (LT2), 27 July 2015 (LT2).

Identification: Deeming (1998).

Known distribution: Undetermined.

\section{Family Pyrgotidae}

Campylocera ferruginea Macquart, 1843

15 November 2015 (LT6).

Identification: Dr Valery Korneyev, from photos (personal communication).

Known distribution: AF.

Eupyrgota latipennis (Walker, 1849)

3 June 2014 (LT2), 14 November 2015 (LT2).

Identification: Dr Valery Korneyev, from photos (personal communication).

Known distribution: AF. 


\section{Family Sphaeroceridae}

Rachispoda fuscipennis (Haliday 1833)

15 February 2014 (PT2, PT3), 23 August 2014 (PT6), 18 October 2014 (LT3, PT1, PT2, PT3, PT4), 8-11 December 2014 (LT2, LT3, LT4, VC1, VC2).

Identification: Magdi El-Hawagry, compared with museum specimens.

Known distribution: PA.

\section{Family Tephritidae}

Subfamily Dacinae

Tribe Dacini

Bactrocera zonata (Saunders, 1842)

23 August 2014 (LT2), 5 May 2015 (SW1), 27 July 2015 (SW1).

Identification: Merz and Dawah ( 2005) and Efflatoun (1924).

Known distribution: OR.

\section{Subfamily Tephritinae}

Tribe Tephritini

Acanthiophilus helianthi (Rossi, 1794)

23 August 2014 (LT2).

Identification: Merz and Dawah ( 2005) and Efflatoun (1924).

Known distribution: AF, OR, PA.

Dioxyna sororcula (Wiedemann, 1830)

15 February 2014 (MT4), 3 June 2014 (MT4), 8 December 2014 (LT5, VC1).

Identification: Merz and Dawah ( 2005) and Efflatoun (1924).

Known distribution: AF.

Goniurellia tridens (Hendel, 1910)

23 August 2014 (LT2).

Identification: Hendel (1910).

Known distribution: PA.

Trupanea stellata (Fuesslin, 1775)

3 June 2014 (LT2).

Identification: Merz and Dawah ( 2005) and Efflatoun (1924).

Known distribution: PA.

\section{Family Ulidiidae}

Subfamily Ulidiinae

\section{Tribe Ulidiini}

Physiphora alceae (Preyssler, 1791)

15 February 2014 (MT1, LT1), 21 April 2014 (LT1), 6 June 2014 (LT1), 23 August 2014 (LT1), 17-18 October 2014 (LT3, PT3), 27 January 2015 (MT1, MT3), 5 May 2015 (LT1), 27 July 2015 (LT1, SW1), 15 November 2015 (LT6, MT4).

Identification: Al Dhafer and El-Hawagry (2016).

Known distribution: Cosmopolitan. 


\section{Subsection Calyptratae}

Family Anthomyiidae

Subfamily Anthomyiinae

Tribe Anthomyiini

Anthomyia pluvialis (Linnaeus, 1758)

15 February 2014 (MT1), 27 January 2015 (MT3), 4-5 May 2015 (MT3, SW1), 15

November 2015 (LT5).

Identification: Michelsen (2008).

Known distribution: PA.

\section{Tribe Hydrophoriini}

Delia platura (Meigen, 1826)

15 February 2014 (LT1, LT2, LT3, MT1), 23 August 2014 (LT2), 17 October 2014 (LT1, LT2), 27 January 2015 (LT2, LT3, MT2).

Identification: Meigen (1826).

Known distribution: Cosmopolitan.

\section{Family Calliphoridae}

Subfamily Calliphorinae

Calliphora croceipalpis Jaennicke, 1867

15 February 2014 (MT4).

Identification: Setyaningrum and Al Dhafer (2014).

Known distribution: AF.

Calliphora vicina (Robineau-Desvoidy, 1830)

3 June 2014 (SW6).

Identification: Setyaningrum and Al Dhafer (2014).

Known distribution: Cosmopolitan.

\section{Subfamily Chrysomyinae}

Chrysomya albiceps (Wiedemann, 1819)

4 June 2014 (SW1), 2 September 2015 (LT6), 15 November (LT3).

Identification: Setyaningrum and Al Dhafer (2014).

Known distribution: AF.

Chrysomya putoria (Wiedemann, 1830)

3 June 2014 (SW4).

Identification: Setyaningrum and Al Dhafer (2014).

Known distribution: AF.

Chrysomya regalis Robineau-Desvoidy, 1830

15 February 2014 (MT3), 4 June 2014 (MT6), 10 December 2014 (LT6).Identification: Setyaningrum and Al Dhafer (2014).

Known distribution: AF. 


\section{Subfamily Luciliinae}

Lucilia sericata (Meigen, 1826)

16 February 2014 (HP6), 21 February 2014 (LT3), 10 December 2014 (LT6).

Identification:

Known distribution: Cosmopolitan.

\section{Subfamily Polleniinae}

Pollenia hungarica Rognes, 1987

17 October 2014 (LT6).

Identification: Setyaningrum and Al Dhafer (2014).

Known distribution: PA.

Pollenia rudis (Fabricius, 1794)

17 October 2014 (LT5).

Identification: Setyaningrum and Al Dhafer (2014).

Known distribution: PA.

\section{Family Muscidae}

Subfamily Atherigoninae

Tribe Atherigonini

Atherigona humeralis Wiedemann, 1830

15 November 2015 (SW5).

Identification: Pont (1991).

Known distribution: AF.

Atherigona laevigata (Loew, 1852)

15 February 2014 (MT1), 8 December 2014 (VC4).

Identification: Pont (1991).

Known distribution: AF.

Atherigona reversura Villeneuve, 1936

15 February 2014 (MT3), 23 August 2014 (LT2, LT3, LT5), 17 October 2014 (LT4, LT5, MT2, MT4), 5 May 2015 (MT2), 15 November 2015 (MT4), 2 September 2015 (LT6).

Identification: Pont (1991).

Known distribution: OR.

\section{Subfamily Coenosiinae}

\section{Tribe Coenosiini}

Coenosia attenuata Stein, 1903

15 February 2014 (MT4, PT4), 23 April 2014 (PT1), 23 August 2014 (LT2), 17 October 2014 (LT2, LT4, MT4), 18 October 2014 (PT5), 5 May 2015 (MT4), 15

November 2015 (MT4).

Identification: Pont (1991).

Known distribution: Cosmopolitan.

Coenosia humilis Meigen, 1826 
5 May 2015 (MT6).

Identification: Pont (1991).

Known distribution: Cosmopolitan.

\section{Tribe Limnophorini}

Lispe nivalis Wiedemann, 1830

15 February 2014 (LT6).

Identification: Pont (1991).

Known distribution: AF.

Lispe pectinipes Becker, 1903

23 August 2014 (LT2, LT3), 17 October 2014 (LT5), 5 May 2015 (LT1, MT2), 14-15

November 2015 (LT4, LT5).

Identification: Pont (1991).

Known distribution: PA.

Subfamily Muscinae

Tribe Muscini

Musca albina Wiedemann, 1830

5 May 2015 (MT6).

Identification: Pont (1991).

Known distribution: AF, OR, PA.

Musca autumnalis De Geer, 1776

23 August 2014 (LT2), 5 May 2015 (MT2).

Identification: Pont (1991).

Known distribution: Cosmopolitan.

Musca calleva Walker, 1849

14 November 2015 (LT4).

Identification: Pont (1991).

Known distribution: AF.

Musca domestica Linnaeus, 1758

15 February 2014 (MT5, PT6), 3 June 2014 (MT2, SW6), 23 August 2014 (LT2, LT3), 5 May 2015 (MT6), 2 September 2015 (LT5), 15 November 2015 (LT6).

Identification: Pont (1991).

Known distribution: Cosmopolitan.

Musca lucidula (Loew, 1856)

3 June 2014 (MT6).

Identification: Pont (1991).

Known distribution: AF, PA.

Musca sorbens Wiedemann, 1830

5 May 2015 (MT1), 15 November 2015 (LT5).

Identification: Pont (1991).

Known distribution: AF. 


\section{Tribe Stomoxyini}

Stomoxys niger Macquart, 1851 Fig. 11

15 February 2014 (MT4), 17 October 2014 (LT5).

Identification: Márcia et al. (2012).

Known distribution: AF. First record from KSA.

\section{Subfamily Phaoniinae}

Tribe Dichaetomyiini

Dichaetomyia luteiventris (Rondani, 1873)

2 March 2015 (PT5).

Identification: Pont (1991).

Known distribution: AF.

\section{Tribe Phaoniini}

Helina coniformis (Stein in Becker, 1903)

15 February 2014 (MT5, PT2), 21 April 2014 (LT2), 17 October 2014 (LT1, LT5, MT1, MT2, MT3, MT4), 27 January 2015 (MT2, MT3), 14-15 November 2015 (LT4, LT5, MT4).

Identification: Pont (1991).

Known distribution: AF.

Helina lucida (Stein, 1913)

21 April 2014 (LT5).

Identification: Pont (1991).

Known distribution: AF.

\section{Family Rhiniidae}

Cosmina viridis Townsend, 1917

15-16 February 2014 (MT1, MT3), 17 October 2014 (LT5), 27 January 2015 (LT1, MT3), 4-5 May 2015 (SW4, MT2).

Identification: Setyaningrum and Al Dhafer (2014).

Known distribution: AF.

Isomyia terminata (Wiedemann, 1830)

15 February 2014 (MT5, PT5).

Identification: Setyaningrum and Al Dhafer (2014).

Known distribution: AF.

Rhinia apicalis (Wiedemann, 1830)

15 February 2014 (MT5), 3 June 2014 (SW4), 17 October 2014 (LT2, LT3, LT5), 14-15 November 2015 (LT4, LT5, LT6).

Identification: Setyaningrum and Al Dhafer (2014).

Known distribution: AF. 


\section{Family Sarcophagidae}

Subfamily Miltogramminae

Taxigramma heteroneura (Meigen, 1830)

15 February 2014 (MT5), 3 June 2014 (SW4), 27 January 2015 (MT4), 5 May 2015

(MT4, SW1), 27-29 July 2015 (PT5).

Identification: Thomas Pape (personal communication) and the first author.

Known distribution: NE, PA.

\section{Subfamily Paramacronychiinae}

Wohlfahrtia erythrocera Villeneuve, 1910

28 July 2015 (PT6).

Identification: Thomas Pape (personal communication) and the first author.

Known distribution: AF.

Wohlfahrtia nuba Wiedemann, 1830

3 May 2015 (PT5).

Identification: Thomas Pape (personal communication) and the first author. Known distribution: AF.

\section{Subfamily Sarcophaginae}

Blaesoxipha algeriensis (Townsend, 1919)

23 August 2014 (LT5).

Identification: Thomas Pape (personal communication) and the first author. Known distribution: PA.

Blaesoxipha rufipes (Macquart, 1839)

3 June 2014 (SW4).

Identification: Thomas Pape (personal communication) and the first author. Known distribution: Cosmopolitan.

Sarcophaga adhamae (Lehrer and Abou-Zied, 2008)

21 April 2014 (BT6).

Identification: Lehrer and Abou-Zied (2008).

Known distribution: AF.

Sarcophaga africa (Wiedemann, 1824)

5 May 2015 (SW4).

Identification: Thomas Pape (personal communication) and the first author. Known distribution: Cosmopolitan.

Sarcophaga babiyari (Lehrer, 1995)

3 June 2014 (LT6).

Identification: Thomas Pape (personal communication) and the first author. Known distribution: AF.

Sarcophaga dux Thompson, 1869

15 February 2014 (MT1).

Identification: Thomas Pape (personal communication) and the first author. Known distribution: Cosmopolitan. 
Sarcophaga palestinensis (Lehrer, 1998) Fig. 12

21 February 2014 (LT1).

Identification: Thomas Pape (personal communication) and the first author.

Known distribution: PA.

\section{Family Tachinidae}

Subfamily Exoristinae

Tribe Eryciini

Drino lota (Meigen, 1824)

15-16 February 2014 (LT6, MT4, MT5, MT6, SW6), 17 October 2014 (LT4, LT5, LT6), 14-15 November 2015 (LT4, LT6).

Identification: Dawah (2011) and Tschorsnig and Herting (1994).

Known distribution: AF, PA.

\section{Tribe Exoristini}

Exorista larvarum (Linnaeus, 1758)

3 June 2014 (SW2, SW4).

Identification: Dawah (2011) and Tschorsnig and Herting (1994).

Known distribution: NE, PA.

\section{Tribe Goniini}

\section{Gonia capitata (De Geer, 1776)}

5 May 2015 (MT1). Identification: Dawah (2011) and Tschorsnig and Herting (1994).

Known distribution: PA.

Sturmia bella (Meigen, 1824)

15 February 2014 (MT1), 21 April 2014 (LT1), 3 June 2014 (SW4), 27-30 January 2015 (LT1, LT2, LT3), 27 July 2015 (LT5).

Identification: Dawah (2011) and Tschorsnig and Herting (1994).

Known distribution: OR, PA.

\section{Subfamily Phasiinae}

\section{Tribe Cylindromyiini}

Cylindromyia bicolor (Olivier, 1812)

7 June 2014 (SW4).

Identification: Dawah (2011), El-Hawagry et al. (2015) and Tschorsnig and Herting (1994).

Known distribution: PA.

\section{Subfamily Tachininae}

\section{Tribe Tachinini}

Dejeania bombylans (Fabricius, 1798)

10 December 2014 (LT6).

Identification: Dawah (2011) and Tschorsnig and Herting (1994).

Known distribution: AF. 
13

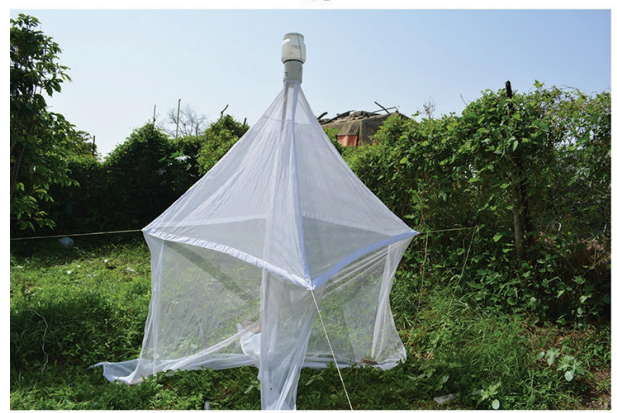

15

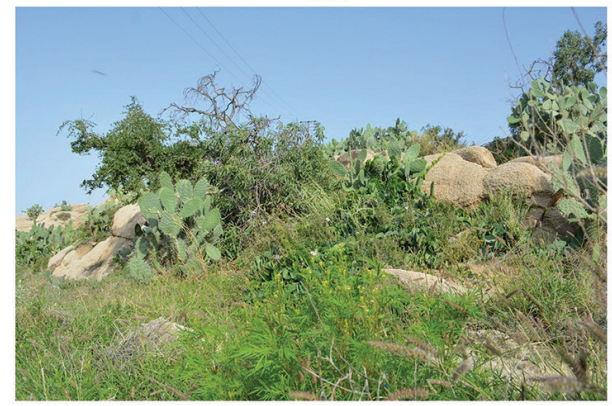

17

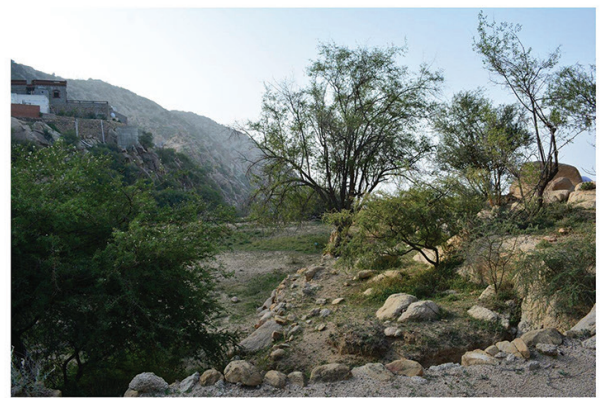

14

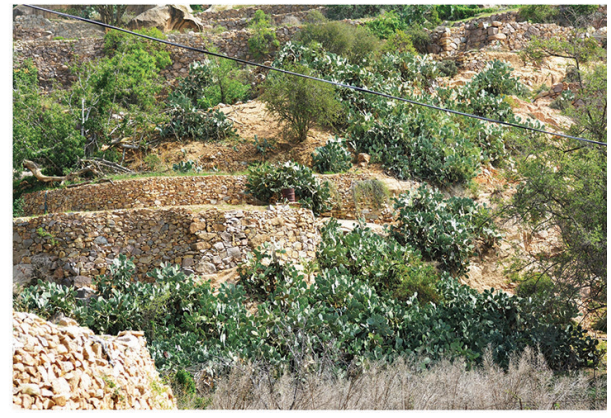

16

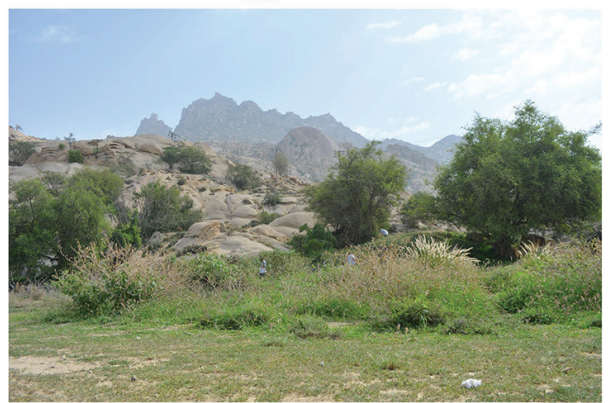

18

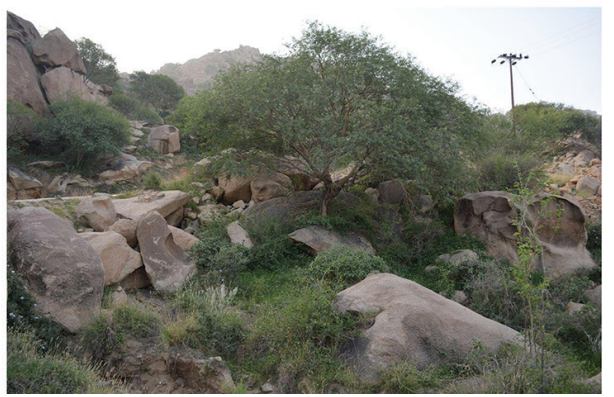

Figures 13-18. 13 Collecting locality no. 1 I4 Collecting locality no. 2 I5 Collecting locality no. 3 16 Collecting locality no. 417 Collecting locality no. 5 I8 Collecting locality no. 6.

\section{Discussion}

In terms of vegetation and speciation, the south-western part of KSA, including $\mathrm{Al}$ Baha Province, is considered to be the most important part of the country and the Arabian Peninsula in general. Floristically and ecologically, this area is similar to the high altitude mountains of north-eastern and eastern parts of Africa, and like other areas in the south-western part of the Arabian Peninsula, contains montane woodlands and evergreen shrub lands, with strong Afromontane affinities (Bussmann and Beck 1995; Zohary 1973; Eig 1938). 
Considering the insect fauna as a whole, El-Hawagry et al. $(2013,2015)$ attributed the extraordinary complex and the interesting insect fauna in Al-Baha Province to its geographical position at the junction of two of the world's main zoogeographical regions, the Afrotropical and the Palaearctic.

Many present day biogeographers think that the biogeographical divisions within the eastern and the northeastern parts of Africa should be extended towards east within the Arabian Peninsula as well, covering the high altitude regions of the southern AlSarawat Mountains, namely "Afromontane Archipelago" (Zohary 1973; Eig 1938). Bolton (1994), Eig (1938), El-Hawagry et al. (2013 and 2015) and Sharaf et al. (2012a, 2012b) concluded that the insect faunal composition in Al-Baha Province has an Afrotropical flavor as the Afrotropical elements were predominantly indicated, they tended to agree with those biogeographers who think that parts of the Arabian Peninsula, including Al-Baha Province, should be included in the Afrotropical region, but they couldn't indicate the northern border of this region exactly. All these facts seem to be reflected somehow on the fly faunal composition in Jabal Shada al-A'la Nature Reserve (SANR) as shown in the present results which obviously emphasize the fact that Al-Baha Province, as lying in the south-western part of the Arabian Peninsula, should be included in the Afrotropical Region rather than in the Palaearctic Region or the Eremic Zone.

\section{Funding}

The authors would like to extend their sincere appreciation to the Deanship of Scientific Research at King Saud University for its funding this research group NO (RGP-1437-009).

\section{Acknowledgements}

We are grateful to Prince Bandar Bin Saud Al Saud, Head of the Saudi National Commission for Wildlife Conservation and Development for the support during the study. We are also indebted to the following people for identifying specimens or providing critical assistance throughout this study: Dr John Deeming, National Museum Wales, Cardiff, Wales, UK; Dr Thomas Pape, Natural History Museum of Denmark, Copenhagen, Denmark; Dr Valery Korneyev, General and Applied Entomology Department, I.I. Schmalhausen Institute of Zoology, National Academy of Sciences of Ukraine; Dr Jason G. H. Londt, Natal Museum, Pietermaritzburg, South Africa; Dr Boris C. Kondratieff, Department of Bioagricultural Sciences and Pest Management, Colorado State University, USA; Dr Neal Evenhuis, Bishop Museum, Honolulu, Hawaii, USA; Dr Babak Gharali, Research Centre for Agriculture and Natural Resources, Iran; Dr Shaun Winterton and Dr Martin Hauser, Plant Pest Diagnostics Branch, California Department of Food \& Agriculture, Sacramento, U.S.A.; Torsten Dikow, Smithsonian Institution, National Museum of Natural History, Washington, USA. Dr Francis Gil- 
bert, School of Life Sciences, University of Nottingham, UK; and Mr Ahmed Shams Al Ola, Plant Protection Department, College of Food and Agriculture Sciences, King Saud University, Saudi Arabia. Sincere thanks go to the research team of the KSMA for collecting and mounting specimens.

\section{References}

Abdullah MA, Al-Mazroui MA (1998) Climatological study of the southwestern region of Saudi Arabia. I. Rainfall analysis. Climate Research 9: 213-223. doi: 10.3354/cr009213 Abdullah MA, Merdan AI (1995) Distribution and ecology of the mosquito fauna in the southwestern Saudi Arabia. Journal of the Egyptian Society of Parasitology 25(3): 815-837.

Alahmed AM, Kheir SM, Al Khereiji MA (2010) Distribution of Culicoides latreille (Diptera: Ceratopogonidae) in Saudi Arabia. Journal of Entomology 7: 227-234. doi: 10.3923/ je.2010.227.234

Al Dhafer HM, El-Hawagry MS (2016) Records for the family Ulidiidae (Diptera, Tephritoidea) in Saudi Arabia.African Entomology. African Entomology 24(1): 225-232. doi: 10.4001/003.024.0225

Alikhan M, Ghamdi KA, Mahyoub JA (2014) Aedes Mosquito Species in Western Saudi Arabia. Journal of Insect Science 14: 69. doi: 10.1673/031.014.69

Amoudi MA (1993) New records of some of Sarcophagid flies with distribution of all knownflesh flies (Diptera, Sarcophagidae) of Saudi Arabia. Journal of the Egyptian Society of Parasitology 23(1): 297-304.

Amoudi MA, Diab FM and Abou-Fannah SSM (1991) Zaprionus indianus Gupta (Diptera, Drosophilidae) in Saudi Arabia and the effect of temperature on the life cycle. Journal of King Saud University 3: 25-35.

Amoudi MA, Leclercq M (1992) The horse flies from Saudi Arabia, distribution and zoogeography (Diptera, Tabanidae). Notes Fauniques de Gembloux 25: 3-15.

Bolton B (1994) Identification guide to the ant genera of the world. Harvard University Press, Cambridge (MA), 232 pp.

Boorman J (1989) Insects of Arabia. Culicoides (Diptera, Ceratopogonidae) of the Arabian Peninsula with notes on their medical and veterinary importance. Fauna of Saudi Arabia, 10: 160-225.

Bussmann RW, Beck E (1995) The forests of Mount Kenia (Kenya), a phytosociological synopsis. Phytocoenologia. 25: 467-560. doi: 10.1127/phyto/25/1995/467

Crosskey RW (1980) Catalogue of the Diptera of the Afrotropical Region. British Museum (Natural History), London, 1437 pp.

Dawah HA (2011) Some Tachinidae (Diptera: Calyptrata) from South-Western Saudi Arabia. Journal of Jazan University - Applied Sciences Branch 1: 28-38.

Dawah HA, Abdullah MA (2006) New Records of Chloropidae (Diptera) from Southwest Saudi Arabia with some Biological Information, World-wide Geographical Distribution and Taxonomic Features. Saudi Journal of Biological Sciences 13(1): 24-34. 
Dawah HA, Abdullah MA (2008) First records of the stalk-eyed flies (Diopsidae: Cyclorrhapha), a pest of Poaceae, from South-Western Saudi Arabia. King Khalid University Journal of Natural, Medical, and Technical Sciences 4: 86-95.

Deeming JC (1998) Milichiidae and Carnidae (Diptera, Cyclorrhapha) from the Arabian Peninsula. Fauna of Saudi Arabia 17: 147-157.

Deeming JC, Al-Dhafer HM (2012) Chloropidae from the Arabian Peninsula (Diptera: Cyclorrhapha). Zoology in the Middle East. 58: 3-88.

Ebejer MJ (2008) Order Diptera, family Chyromyidae. In: Harten A van (Ed.) Arthropod fauna of the United Arab Emirates (Vol. 1). Dar Al Ummah Printing, Abu Dhabi, 683-695.

Efflatoun HC (1924) A monograph of Egyptian Diptera, Part II., Fam. Trypaneidae. Mémoires de la Société Royale Entomologique d'Egypte 2(2): 1-132.

Efflatoun HC (1934) A monograph of Egyptian Diptera. Part IV, Family Asilidae (Section I). Mémoires de la Société Royale Entomologique d'Egypte 4(2): 1-198.

Efflatoun HC (1937) A monograph of Egyptian Diptera. Part IV, Family Asilidae (Section II). Mémoires de la Société Royale Entomologique d'Egypte 4(3): 199-443.

Eig A (1938) Taxonomic Studies on the Oriental Species of the Genus Anthemis. Palestine. Journal of Botany, Jerusalem 1: 161-224.

El-Hawagry MS, Greathead DJ (2006) Review of the genus Villa Lioy (Bombyliidae, Diptera) from Egypt, with descriptions of two new species. Zootaxa 1113: 21-32.

El-Hawagry M (2015) Catalogue of Superfamily Asiloidea. Lap Lambert Academic Publishing, Saarbrücken, Germany, 196 pp.

El-Hawagry MS, Al Dhafer HM (2015) Five new records of bee flies (Bombyliidae, Diptera) from Saudi Arabia with zoogeographical remarks. ZooKeys 489: 125-133. doi: 10.3897/ zookeys.489.8794

El-Hawagry MS, El-Moursy AA, Gilbert F, Zalat S (2000) The Tribe Anthracini Latreille (Bombyliidae, Diptera) from Egypt. Egyptian Journal of Biology 2: 97-117.

El-Hawagry MS, Evenhuis NL (2008) An updated key to the genera of Egyptian beeflies (Diptera: Bombyliidae). Egyptian Journal of Biology 10: 104-121.

El-Hawagry MS, Gilbert F (2014) Zoogeographical affinities and faunal relationships of bee flies (Diptera: Bombyliidae) in Egypt. Zoology in the Middle East 60:1, 50-56. doi: 10.1080/09397140.2014.892339

El-Hawagry MS, Khalil MW, Sharaf MR, Fadl HH, Aldawood AS (2013) A preliminary study on the insect fauna of Al-Baha Province, Saudi Arabia, with descriptions of two new species. ZooKeys 274: 1-88. doi: 10.3897/zookeys.274.4529

El-Hawagry MS, Sharaf MR, Al Dhafer HM, Fadl HH, Aldawood AS (2015) Addenda to the insect fauna of Al-Baha Province, Kingdom of Saudi Arabia with zoogeographical notes. Journal of Natural History 50(19/20): 1209-1236. doi: 10.1080/00222933.2015.1103913

El-Juhany LI, Aref IM (2013) The Present Status of the Natural Forests in the Southwestern Saudi Arabia: 3- Asir and East Jazan Forests. World Applied Sciences Journal 21(5): 710-726. doi: 10.5829/idosi.wasj.2013.21.5.2841

Evenhuis NL, Greathead DJ (2015) [online] World catalog of bee flies (Diptera: Bombyliidae). http:// hbs.bishopmuseum.org/bombcat/bombcat-revised2015.pdf [accessed in April and May 2016] Ghazanfar, SA, Fisher M (1998) Vegetation of the ArabianPeninsula. Geobotany 25: 1-362. 
Glick JI (1992) Illustrated Key to the Female Anopheles of Southwestern Asia and Egypt (Diptera: Culicidae). Mosquito Systematics 24(2): 125-153.

Greathead DJ (1980) Diptera, Fam. Bombyliidae. Fauna Saudi Arabia 2: 291-337.

Greathead DJ (1988) Diptera, Fam. Bombyliidae of Saudi Arabia (part 2). Fauna of Saudi Arabia 9: 90-113.

Grichanov IY (2007) A checklist and keys to Dolichopodidae (Diptera) of the Caucasus and East Mediterranean. VIZR RAAS, St.Petersburg, 160 pp. [Plant Protection News, Supplement]

Haenni JP (1985) Diptera: Fam. Bibionidae and Scatopsidae of Saudi Arabia. Fauna of Saudi Arabia 7: 198-20.

Hegazy AK, El Demerdash MA, Hosni HA (1998) Vegetation, species diversity and floristic relations along an altitudinal gradient in south-west Saudi Arabia. Journal of Arid Environments 38: 3-13. doi: 10.1006/jare.1997.0311

Hendel F (1910) Uber acalyptrate Musciden. Wiener Entomologische Zeitung 29: 101-127.

Hölzel H (1998) Zoogeographical features of Neuroptera of the Arabian Peninsula. Acta Zoologica Fennica 209: 129-140.

Ibrahim AA, Abdoon MA (2005) Distribution and Population Dynamics of Phlebotomus Sandflies (Diptera: Psychodidae) in an Endemic Area of Cutaneous leishmaniasis in Asir Region, Southwestern Saudi Arabia. Journal of Entomology 2: 102-108. doi: 10.3923/ je.2005.102.108

Kirk-Spriggs AH, McGregor G (2009) Disjunctions in the Diptera (Insecta) fauna of the Mediterranean Province and southern Africa and a discussion of biogeographical considerations. Transactions of the Royal Society of South Africa 64: 32-52. doi: 10.1080/00359190909519236

Lamb CG (1918) Notes on exotic Chloropidae - Part II Oscininae [part]. Annals \& Magazine of Natural History 9: 329-348. doi: 10.1080/00222931808562325

Larsen TB (1984) Butterflies of Saudi Arabia and its neighbours. Stacey International, London, $160 \mathrm{pp}$.

Leclercq M (1982) Diptera, family Tabanidae of Saudi Arabia. Fauna of Saudi Arabia 4: 447-449.

Leclercq M (1986) Diptera, family Tabanidae of Saudi Arabia (part2). Fauna of Saudi Arabia 8: 340-342.

Leclercq M (2000) A faunistic account of Tabanidae (Diptera) of Saudi Arabia and Oman. Fauna of Arabia 18: 285-292.

Lehrer AZ, Abou-Zied EM (2008) Une espece nouvelle du genre Engelisca Rohdendorf de la faune d'Arabie Saoudite (Diptera, Sarcophagidae). Fragmenta Dipterologica 14: 1-4.

Lewanczyk A, Szadziewski R, Dominiak P (2009) Diagnosis of Forcipomyia sahariensis Kieffer (Diptera: Ceratopogonidae) with the first description of immature stages. Fragmenta Faunistica 52: 149-155. doi: 10.3161/00159301FF2009.52.2.149

Londt JGH (2008) A review of Afrotropical Trichardis Hermann, 1906, and the description of the first Oriental representative of the genus (Diptera: Asilidae: Laphriinae). African Invertebrates 49(2): 171-226. doi: 10.5733/afin.049.0210

MacGowan I, Friedberg A (2009) The Lonchaeidae of Israel (Diptera), with a description of three new species. Israel Journal of Entomology 38: 61-92. 
Márcia SC, Claudio JBC, Pont AC (2012) Taxonomy of the Muscidae (Diptera) of Namibia: a key to genera, diagnoses, new records and description of a new species. African Invertebrates 53(1): 47-67. doi: 10.5733/afin.053.0103

McAlpine JF (1981) Manual of Nearctic Diptera. Research Branch, Agriculture Canada, Ottawa. Mei M, Stuke J-H (2008) Remarks on Zodion nigritarsis (Strobl, 1902) and other European species of Zodion Latreille, 1796, with a revised key (Diptera, Conopidae). Tijdschrift voor Entomologie 151: 3-10. doi: 10.1163/22119434-900000247

Meigen JW (1826) Systematische Beschreibung der bekannten europaischen zweiflugeligen Insekten. Funfter Theil. Schulz-Wundermann, Hamm, 412 pp.

Merz B, Dawah H (2005) Fruit flies (Diptera, Tephritidae) from Saudi Arabia, with descriptions of a new genus and six new species. Revue Suisse de Zoologie, 983-1028. doi: 10.5962/bhl.part.80334

Michelsen V (2008) The Anthomyia pluvialis complex in Europe (Diptera, Anthomyiidae). Systematic Entomology 5(3): 281-290. doi: 10.1111/j.1365-3113.1980.tb00416.x

Mohrig W, Heller K, Hippa H, Vilkamaa P, Menzel F (2012) Revision of black fungus gnats (Diptera: Sciaridae) of North America. Studia Dipterologica 19: 141-286.

Narchuk EP (2007) Nemestrinid flies (Diptera, Nemestrinidae) in the fauna of Eastern Europe and the Caucasus. Entomological Review 87: 1076. doi: 10.1134/S0013873807080143

Pape T (1996) A catalogue of the Sarcophagidae of the world (Insecta: Diptera). Memoirs on Entomology, International 8: 1-558.

Pape T, Thompson FC (Eds) (2016) Systema Dipterorum. Version 2.0. http://www.diptera.org [accessed in May 2016]

Papp L (1978) Species of nine acalyptrate fly families from Tunisia. Folia entomologica hungarica 31: 2000-2001.

Pont AC (1991) A review of the Fanniidae and Muscidae of the Arabian Peninsula. Fauna of Saudi Arabia 12: 312-365.

Sabrosky CW (1987) Milichiidae. In: McAlpine JF (Ed.) Manual of Nearctic Diptera. Research Branch, Agriculture Canada, Ottawa, v. 2: Monograph, n. 28, 903-908.

Sclater PL (1858) On the general geographical distribution of the class Aves. Journal of the Proceedings of the Linnean Society: Zoology 2: 130-145. doi: 10.1111/j.1096-3642.1858.tb02549.x

Setyaningrum H, Al Dhafer HM (2014) The Calliphoridae the blow flies (Diptera: Oestroidea) of Kingdom of Saudi Arabia. Egyptian Academic Journal of Biological Sciences 7: 49-139. Sharaf MR, Aldawood AS, El-Hawagry MS (2012a) A new ant species of the genus Tapinoma (Hymenoptera, Formicidae) from Saudi Arabia with a key to the Arabian species. ZooKeys 212: 35-43. doi: 10.3897/zookeys.212.3325

Sharaf MR, Aldawood AS, El-Hawagry MS (2012b) First record of the ant subfamily Aenictinae (Hymenoptera, Formicidae) from Saudi Arabia, with the description of a new species. ZooKeys 228: 39-49. doi: 10.3897/zookeys.228.3559

Soós Á, Papp L (Eds) (1984-1993) Catalogue of Palaearctic Diptera (14 vols) Akademiai Kiado and Hungarian Natural history Museum, Budapest and Elsevier Science, Amsterdam.

Steffan WA (1981) Sciaridae. In: McAlpine JF, Peterson BV, Shewell GE, Teskey HJ, Vockeroth JR, Wood DM (Eds) Manual of Nearctic Diptera. Research Branch Agriculture Canada, Monograph 27, 247-255. 
SWA (2016) Protected Areas. Saudi Wildlife Commission. http://www.swa.gov.sa [accessed in June 2016]

Tschorsnig H-P, Herting B (1994) The Tachinids (Diptera: Tachinidae) of Central Europe: Identification Keys for the Species and Data on Distribution and Ecology. State Museum of Natural Science, Stuttgart, 150 pp.

Thielman AC, Hunter FF (2007) A Photographic Key to Adult Female Mosquito Species of Canada (Diptera: Culicidae). Canadian Journal of Arthropod Identification 4: 1-117.

Thomas J (2016) Vegetation: Asir Mountains. Plant Diversity in Saudi Arabia. http://plantdiversityofsaudiarabia.info/index.htm [accessed in May 22, 2016]

UAEinteract (2016) Terrestrial mammals. http://www.uaeinteract.com/uaeint_misc/ teanh/025tmam.pdf [accessed in October, 2016]

Unwin DM (1991) A key to the families of British Diptera. http://www.mydiptera.webege. com/Brachycera/Brachycera.html [accessed in April and May 2016]

Uvarov BP (1938) Ecological and biogeographical relations of Eremian Acrididae. Mémoires de la Société de Biogéographie de Paris 6: 231-273.

Wallace AR (1876) The geographical distribution of animals. Mac Millan, London, 607 pp.

Wiedemann CRW (1824) Munus rectoris in Academia Christiana Albertina aditurus analecta entomologica ex Museo Regio Havniensi maxime congesta profert iconibusque illustrat.. Regio typographeo scholarum, Kiliae [= Kiel], 60 pp. doi: 10.5962/bhl.title.77322

Wiedemann CRW (1830) Aussereuropaische zweiflugelige Insekten. Zweiter Theil. Schulz, Hamm, 684 pp.

Zohary M (1973) Geobotanical foundations of the Middle East, vols. 1-2. Fischer, Stuttgart, Amsterdam, Swets, Zeitlinger, 738 pp. 